\title{
Optimization and antiviral analysis of peptide ligands for the HIV-I packaging signal PSI
}

\author{
Julia Dietz ${ }^{1}$, Anette Pustowka ${ }^{1}$, Ajit Kaur ${ }^{2}$, Joachim Koch ${ }^{3}$, Sarah Mensch ${ }^{2}$, \\ Stefan Stein ${ }^{1}$, Manuel Grez ${ }^{1}$, Gilles Divita ${ }^{4}$, Yves Mély ${ }^{5}$, Harald Schwalbe ${ }^{2}$ \\ and Ursula Dietrich*1
}

\begin{abstract}
Address: ${ }^{1}$ Georg-Speyer-Haus, Frankfurt/Main, Germany, ${ }^{2}$ Organic Chemistry, J. W. Goethe University, Frankfurt, Germany, ${ }^{3}$ Biochemistry, J. W. Goethe University, Frankfurt, Germany, ${ }^{4}$ CRBM-CNRS, Montpellier, France and ${ }^{5}$ CNRS, Université Louis Pasteur, Strasbourg, France

* Corresponding author
\end{abstract}

from 2006 International Meeting of The Institute of Human Virology

Baltimore, USA. 17-2I November, 2006

Published: 21 December 2006

Retrovirology 2006, 3(Suppl I):S58 doi:I0.II86/I742-4690-3-SI-S58

(C) 2006 Dietz et al; licensee BioMed Central Ltd.

\section{Background}

We selected peptide ligands for the HIV-1 packaging signal PSI by screening phage displayed peptide libraries. Peptide ligands were optimized by screening spot synthesis peptide membranes. The aim of this study is the functional characterization of these peptide ligands with respect to inhibition of HIV-1 replication.

\section{Methods}

Phage displayed peptide libraries were screened with PSIRNA structures. The Trp-rich peptide motifs were optimized for specific binding on spot synthesis peptide membranes. The best binding peptide was expressed intracellularly in fusion with RFP or linked to a protein transduction domain (PTD) for intracellular delivery. The effects on virion production were analyzed using pseudotyped lentiviral particles.

\section{Results}

After positive and negative selection rounds, phages binding specifically to PSI-RNA were identified by ELISA. Peptide inserts contained conserved motifs of aromatic amino acids known to be implicated in binding of PSI-RNA by the natural Gag ligand. The filter assay identified HKWPWW as the best binding ligand for PSI-RNA, which is delivered into several cell lines by addition of a PTD. Compared to a control peptide, the HKWPWW peptide inhibited HIV-1 replication as deduced from reduced tit- ers of culture supernatants. As HKWPWW also binds to the TAR-RNA like the natural nucleocapsid PSI-RNA ligand, the effect on Tat-TAR inhibition will also be analyzed. Currently T-cell lines are established which stably express HKWPWW as well as a control peptide, which will be infected with HIV-1 to monitor the ability of HKWPWW to inhibit wild type HIV-1 replication.

\section{Conclusion}

The selection of a peptide ligand for PSI-RNA able to inhibit HIV-1 replication proves the suitability of the phage display technology for the selection of peptides binding to RNA-structures. This enables the indentification of peptides serving as leads to interfere with additional targets in the HIV-1 replication cycle.

\section{Acknowledgements}

This work is supported by the SFB579 "RNA-ligand interactions" and the EU TRloH program. 